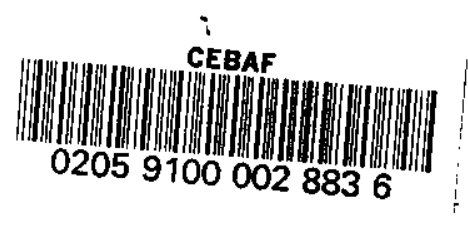

\title{
NONLOCAL CONDENSATES AND QCD SUM RULES FOR THE PION FORM FACTOR ${ }^{1}$
}

\author{
A.P.BAKULEV \\ Physics Department, VINITI, Moscow, USSR \\ A.V.RADYUSHKIN \\ Continuous Electron Beam Accelerator Facility, \\ Newport News, VA 23606, USA \\ and \\ Laboratory of Theoretical Physics, JINR, Dubna, USSR ${ }^{2}$
}

\footnotetext{
${ }^{1}$ This work was supported in part by the U.S.Department of Energy under contract DE-AC0584ER 40150

${ }^{2}$ Address after July 16, 1991; E-mail radyush@icebaf, radyush@theor.jinrc.dubna.su
} 


\section{$\because \quad$ Abstract}

We extend the QCD sum rule analysis of the pion electromagnetic form factor $F_{\pi}\left(Q^{2}\right)$ into the region of moderately large momentum transfers $3 \mathrm{GeV}^{2} \lesssim Q^{2} \lesssim 10 \mathrm{GeV}^{2}$, where one should take into account the effects due to nonzero average virtuality of the vacuum quarks and. gluons. 
1.Introductory remarks. Though the perturbative QCD is undoubtedly an adequate tool to study the asymptotic $Q^{2} \rightarrow \infty$ behavior of hard exclusive reactions $[1,2,3,4,-5]$, there is still no agreement about whether the accessible momentum transfers are large enough to be treated as the asymptotic ones. There is a belief $[6,7]$ that one can apply the perturbative QCD approach for the pion form factor at $Q^{2}>3-4 \mathrm{GeV}^{2}$ by calculating the hard-gluon exchange diagram and using the "camel"-type pion wave function proposed by Chernyak and Zhitnitsky [8]. The nonperturbative soft contribution is assumed to be negligibly small above $3 \mathrm{GeV}^{2}$. Our point of view [9] (coinciding with that of Isgur and Llewellyn-Smith [10]) is precisely the opposite one: we claim that the soft contribution itself is sufficiently large to describe the data, while the one-gluon exchange diagram can be treated as a small $O\left(\alpha_{0} / \pi\right)$ radiative correction. This statement is based on the QCD sum rule calculation of the pion form factor at intermediate $\left(0.5 \mathrm{GeV}^{2} \lesssim Q^{2} \lesssim 3 \mathrm{GeV}^{2}\right)[11,12]$ and small $\left(0<Q^{2}<0.5 \mathrm{GeV}^{2}\right)$ [13] momentum transfers. However, it is very desirable to support it also by an analysis of the soft and hard contributions for higher $Q^{2}$. Our goal in the present paper is to extend the QCD sum rule analysis of refs. $[11,12]$ into the region of moderately large momentum transfers $3 \mathrm{GeV}^{2} \lesssim Q^{2} \lesssim 10 \mathrm{GeV}^{2}$.

2. $Q C D$ sum rules for the pion form factor. Among existing approaches to the analysis of the nonperturbative effects in QCD the most close to perturbative QCD is the QCD sum rule method [15]. To apply it to the pion form factors one should consider both the operator product expansion

$$
T\left(p_{1}^{2}, p_{2}^{2}, q^{2}\right)=T^{\text {pert }}\left(p_{1}^{2}, p_{2}^{2}, q^{2}\right)+c_{1} \frac{\langle G G\rangle}{\left(p^{2}\right)^{3}}+c_{2} b \frac{\alpha_{0}\langle\bar{q} q\rangle^{2}}{\left(p^{2}\right)^{4}}+\ldots
$$

and the dispersion relation

$$
T\left(p_{1}^{2}, p_{2}^{2}, q^{2}\right)=\frac{1}{\pi^{2}} \int_{0}^{\infty} d s_{1} \int_{0}^{\infty} d s_{2} \frac{\rho\left(s_{1}, s_{2}, q^{2}\right)}{\left(s_{1}-p_{1}^{2}\right)\left(s_{2}-p_{2}^{2}\right)}+\text { "subtractions" }
$$

for the relevant three-point function $T\left(p_{1}^{2}, p_{2}^{2}, q^{2}\right)$ calculated at sufficiently large spacelike values of the momenta $p_{1}, p_{2}$ corresponding to the local currents with the pion quantum numbers (we take the axial current $j_{5}^{\alpha}=\bar{d} \gamma_{5} \gamma^{\alpha} u$, its projection onto the pion state $|P, \pi\rangle$ being proportional to the pion decay constant $\left.f_{\pi}:\left\langle 0\left|j_{6}^{\alpha}\right| P, \pi\right\rangle=i f_{\pi} P^{\alpha}\right)$. A similar dispersion representation (with the same subtraction terms) holds for the perturbative term $T^{\text {pert }}\left(p_{1}^{2}, p_{2}^{2}, q^{2}\right)$. The relevant spectral density

$$
\rho^{\text {pert }}\left(s_{1}, s_{2}, Q^{2}\right)=\frac{3}{2}\left[\frac{\left(Q^{2}\right)^{2}}{2 !}\left(\frac{d}{d Q^{2}}\right)^{2}+\frac{\left(Q^{2}\right)^{3}}{3 !}\left(\frac{d}{d Q^{2}}\right)^{3}\right] \frac{1}{\sqrt{\left(s_{1}+s_{2}+Q^{2}\right)^{2}-4 s_{1} s_{2}}}
$$

is a smooth function describing the transitions between free-quark $\vec{u} d$-states with invariant masses $s_{1}$ and $s_{2}$, respectively. It differs, of course, from the physical (hadronic) spectral density $\rho\left(s_{1}, s_{2}, Q^{2}\right)$ that contains the term corresponding to the pion elastic form factor $\rho_{\pi \pi}\left(s_{1}, s_{2}, Q^{2}\right)=\pi^{2} f_{\pi}^{2} F_{\pi}\left(Q^{2}\right) \delta\left(s_{1}-m_{\pi}^{2}\right) \delta\left(s_{2}-m_{\pi}^{2}\right)$ and the contributions corresponding to transitions involving higher resonances. The difference between $\rho\left(s_{1}, s_{2}, q^{2}\right)$ and 
$\rho^{\text {pert }}\left(s_{1}, s_{2}, q^{2}\right)$ is reflected by the nonperturbative contributions to $T\left(p_{1}^{2}, p_{2}^{2}, q^{2}\right)$ proportional to quark and gluon condensates $\langle\bar{q} q\rangle,\langle G G\rangle$ etc.

The model spectral density $\rho\left(s_{1}, s_{2}, q^{2}\right)$ should give the best possible agreement between the two expressions for $T$ (eqs. (1) and (2)). The simplest approximation is to assume that higher states contributions are reproduced by the perturbative spectral density

$$
\rho\left(s_{1}, s_{2}, Q^{2}\right)=\rho_{\pi \pi}\left(s_{1}, s_{2}, Q^{2}\right)+\left(1-\theta\left(s_{1}<s_{0}\right) \theta\left(s_{2}<s_{0}\right)\right) \rho^{\text {pert }}\left(s_{1}, s_{2}, Q^{2}\right),
$$

with $s_{0}$ being the effective threshold for the higher states production. Normally, $s_{0}$ corresponds to the midpoint between the lowest state and the first resonance. Since the $A_{1}$-mass ${ }^{2}$ is $1.6 \mathrm{GeV}^{2}$, one should expect that $s_{0}$ in our case should be about $0.8 \mathrm{GeV}^{2}$.

Combining the two expressions and using the Borel transformation [15] (it produces an exponential weight in the dispersion integral) one obtains the QCD sum rule

$f_{\pi}^{2} F_{\pi}\left(Q^{2}\right)=\frac{\dot{1}}{\pi^{2}} \int_{0}^{s_{0}} d s_{1} \int_{0}^{s_{0}} d s_{2} \rho^{\text {pert }}\left(s_{1}, s_{2}, Q^{2}\right) \exp \left(-\frac{s_{1}+s_{2}}{2 M^{2}}\right)+a \frac{\alpha_{0}\langle G G\rangle}{24 \pi M^{2}}+b \frac{16 \pi \alpha_{1}\langle\bar{q} q\rangle^{2}}{81 M^{4}}+\ldots$

with the coefficients $a$ and $b$ specified by the OPE.

Then, performing a fitting procedure, one finds the value of $s_{0}$ that provides the most stable result for $f_{\pi}^{2} F_{\pi}\left(Q^{2}\right)$ in the widest possible range of the auxiliary Borel parameter $M^{2}$. There exists a correlation between the value of $f_{\pi}^{2} F_{\pi}\left(Q^{2}\right)$ in the stability region and the relevant parameter $s_{0}$. To a good accuracy, it can be described by the (local) duality relation, following from the SR (5) in the formal $M^{2} \rightarrow \infty$ limit

$$
\begin{array}{r}
F_{\pi}^{L D}\left(Q^{2}\right)=\frac{1}{\pi^{2}} \int_{0}^{s_{0}} d s_{1} \int_{0}^{s_{0}} d s_{2} \rho^{\text {pert }}\left(s_{1}, s_{2}, Q^{2}\right) \exp \left(-\frac{s_{1}+s_{2}}{2 M^{2}}\right)= \\
=\frac{s_{0}}{4 \pi^{2} f_{\pi}^{2}}\left[1-\frac{1+6 s_{0} / Q^{2}}{\left(1+4 s_{0} / Q^{2}\right)^{3 / 2}}\right] .
\end{array}
$$

The condensates are formally absent in this relation. However, the value of the duality interval $s_{0}$, extracted from the fitting procedure, is determined by their magnitude, or more precisely, by the ratio of the condensate contribution to the perturbative term.

The form of the $1 / p^{2}$ expansion (OPE) for the three-point amplitudes $T\left(p_{1}^{2}, p_{2}^{2}, q^{2}\right)$ depends on the interrelation between $q^{2}=-Q^{2}<0$ and $\left|p^{2}\right|$. The simplest, symmetric situation $\left|p_{1}^{2}\right| \sim\left|p_{2}^{2}\right| \sim Q^{2}$ (intermediate $Q^{2}$ ) was studied in refs. $[11,12\}$. In this case $a=1, b=\frac{1}{4}\left(13+\frac{Q^{2}}{M^{2}}\right)$. It is instructive to compare these values to those present in the QCD sum rule for the pion decay constant [15]:

$$
f_{\pi}^{2}=\frac{M^{2}}{4 \pi^{2}}\left(1-e^{-\lrcorner_{0} / M^{2}}\right)+\frac{\alpha_{s}\langle G G\rangle}{12 \pi M^{2}}+11 \frac{16 \pi \alpha_{s}\langle\bar{q} q\rangle^{2}}{81 M^{4}} .
$$

Thus, the sum rule for $f_{\pi}^{2} F_{\pi}\left(Q^{2}\right)$ is similar to that for $f_{\pi}^{2}$, but the relevant $\langle G G\rangle$-term is smaller by factor 2 and the $\alpha_{0}\langle\bar{q} q\rangle^{2}$-term is smaller by factor $\sim 3.5$. This suggests that the value $s_{0} \approx 4 \pi^{2} f_{\pi}^{2}$ dictated by the $f_{\pi}^{2}$ sum rule will be reproduced by the form factor sum rule 
only in some intermediate $Q^{2}$-region where $F_{\pi}\left(Q^{2}\right)$ varies between 0.5 and 0.3 , i.e., for $Q^{2}$ between $0.5 \mathrm{GeV}^{2}$ and $1 \mathrm{GeV}^{2}$. This suggestion is supported by an explicit fitting.procedure: $s_{0}=0.7 \mathrm{GeV}^{2}$ for $Q^{2}=0.5 \mathrm{GeV}^{2}$ and $s_{0}=0.9 \mathrm{GeV}^{2}$ for $Q^{2}=1 \mathrm{GeV}^{2}$. For higher $Q^{2}$, the fitting gives larger values: $s_{0}=1.0 \mathrm{GeV}^{2}$ for $Q^{2}=1.5 \mathrm{GeV}^{2}$, while for $Q^{2} \lesssim 0.5 \mathrm{GeV}^{2}$ one would formally obtain values smaller than $0.7 \mathrm{GeV}^{2}$. However, in the region of small $Q^{2}$, one should use a modified form of the OPE [13], containing extra terms which vanish as $Q^{2}$ increases. In particular, for $Q^{2}=0$, the condensate contributions in the form factor sum rule coincide with those in the $f_{\pi}^{2}$ sum rule (this consequence of the Ward identity insures that $F_{\pi}(0):=1$ ) and then decrease to the values obtained in the symmetric kinematics, e.g., $a=\left(1+\frac{m_{p}^{2}}{m_{\rho}^{2}+Q^{2}}\right)$. Hence, in the small- $Q^{2}$ region, the condensate contributions decrease together with the perturbative term, and, as a result, the scale so remains more or less constant and close to the original value $s_{0} \approx 4 \pi^{2} f_{\pi}^{2}$.

In ref.[12], it was demonstrated that the local duality prescription (6), with the constant value $s_{0}=4 \pi^{2} f_{\pi}^{2} \approx 0.7 \mathrm{GeV}^{2}$, gives the contribution sufficiently large to describe the data up to $Q^{2} \sim 4 \mathrm{GeV}^{2}$. With so growing as $Q^{2}$ increases, one gets even larger contributions. However, a universal, $Q^{2}$-independent value for $s_{0}$ is more natural $[12,16,9]$. In particular, the local duality formula $(6)$ in this case allows for an interpretation in terms of the effective light-cone wave functions $\psi\left(x, k_{\perp}\right) \sim \theta\left(k_{\perp}^{2}<x(1-x) s_{0}\right)$.

On the other hand, the QCD sum rule (5) apparently indicates that the scale $s_{0}$ should be a growing function of $Q^{2}$ because, when $Q^{2}$ increases, the perturbative term decreases (eventually, as $1 / Q^{4}$ for large $Q^{2}$ ), whereas the condensate contributions remain constant, with the quark term even growing. The ratio of the condensate terms to the perturbative one increases and, as a result, all the hadronic scales induced by the (effectively increasing) condensate contributions are also growing with $Q^{2}$.

The fact that the condensate contributions in the form factor sum rule are constant or even growing with $Q^{2}$ is surprizing, since the form-factor-type diagrams should generate the contributions decreasing as $Q^{2}$ increases. But the diagrams producing the condensate contributions differ from the ordinary diagrams: some propagators are substituted by constant factors, e.g., quark propagator $\langle T(q(z) \vec{q}(0))\rangle$ is substituted by the quark condensate $\langle\bar{q}(0) q(0)\rangle$. As a result, instead of a $Q^{2}$-dependent contribution one obtains a constant one. The dependence on $Q^{2}$ appears when one calculates the contributions of the higher-dimension operators $\left\langle\bar{q}(0) D^{2} q(0)\right\rangle,\left\langle\bar{q}(0)\left(D^{2}\right)^{2} q(0)\right\rangle$ etc., produced by the Taylor expansion of the original nonlocal condensate $\langle\bar{q}(0) q(z)\rangle$, the nonperturbative part of the quark propagator. The resulting total condensate contribution decreases for large $Q^{2}$. Each term of the standard OPE, however, has the structure $\left(Q^{2} / M^{2}\right)^{n}$, and one should resum them to get a meaningful result. Our strategy is to avoid the original Taylor expansion and deal directly with the nonlocal condensates. This leads to a modified diagram technique, with some lines and vertices corresponding to the nonlocal condensates.

3. Nonlocal condensates [14]. At the two-loop level, to which we restrict our analysis here, one needs bilocal quark and gluon condensates, trilocal quark-gluon condensates and four-quark condensates. For explicit calculations, it is convenient to parametrize the bilocal 
condensates a la the well-known $\alpha$-representation for propagators:

$$
\begin{aligned}
& M(z) \equiv\langle\bar{q}(0) q(z)\rangle=\langle\bar{q}(0) q(0)\rangle \int_{0}^{\infty} e^{\nu z^{2} / 4} f_{S}(\nu) d \nu, \\
& M_{\mu}(z) \equiv\left\langle\bar{q}(0) \gamma_{\mu} q(z)\right\rangle=-i z_{\mu} A \int_{0}^{\infty} e^{\nu z^{2} / 4} f_{V}(\nu) d \nu,
\end{aligned}
$$

where $A=\frac{2}{81} \pi \alpha_{s}\langle\bar{q} q\rangle^{2}$. The trilocal condensates

$$
\begin{aligned}
& M_{\mu \nu}(y, z) \equiv\left\langle\bar{q}(0) \gamma_{\nu} A_{\mu}(y) q(z)\right\rangle=\left(z_{\mu} y_{\nu}-g_{\mu \nu}(z y)\right) M_{1}+\left(y_{\mu} y_{\nu}-g_{\mu \nu} y^{2}\right) M_{2}+\ldots, \\
& \bar{M}_{\mu \nu}(y, z) \equiv\left\langle\bar{q}(0) \gamma_{\nu} \gamma_{5} A_{\mu}(y) q(z)\right\rangle=\epsilon_{\mu \nu \rho \sigma} y^{\rho} z^{\sigma} M_{3}+\ldots
\end{aligned}
$$

can be parameterized by a triple integral representation of the same type:

$$
M_{i}\left(z^{2}, y^{2},(z-y)^{2}\right)=A_{i} \int_{0}^{\infty} e^{\nu_{1} z^{2} / 4+\nu_{2} y^{2} / 4+\nu_{3}(z-y)^{2} / 4} f_{i}\left(\nu_{1}, \nu_{2}, \nu_{3}\right) d \nu_{1} d \nu_{2} d \nu_{3}
$$

where $A_{i}=\left\{-\frac{3}{2} A, 2 A, \frac{3}{2} A\right\}$. The four-quark condensates are factorized via the vacuum dominance hypothesis into a product of two bilocal quark condensates.

All fields are taken in the Fock-Schwinger gauge $z^{\mu} A_{\mu}(z)=0$ where the path-ordered exponentials (required by gauge invariance) are equal to 1 and the covariant derivatives coincide with the ordinary ones.

Expanding the nonlocal condensates into a Taylor series over the local ones is equivalent to an expansion of $f(\nu)^{\prime}$ 's over $\delta^{(n)}(\nu)$. In the limiting case of the standard local condensates one has $f_{S}=\delta(\nu), f_{V}=\delta^{\prime}(\nu), f_{i}\left(\nu_{1}, \nu_{2}, \nu_{3}\right)=\delta\left(\nu_{1}\right) \delta\left(\nu_{2}\right) \delta\left(\nu_{3}\right)$. Including the next term gives, e.g., $f_{S}(\nu)=\delta(\nu)-\frac{1}{2} \lambda_{q}^{2} \delta^{\prime}(\nu)+\ldots$, with

$$
\lambda_{q}^{2} \equiv \frac{\left\langle\bar{q} D^{2} q\right\rangle}{\langle\bar{q} q\rangle}
$$

being the parameter characterizing average virtuality of the vacuum quarks. It is inversely proportional to the correlation length of the nonperturbative vacuum fluctuations. Retaining only the lowest term of the Taylor expansion amounts to the assumption that this correlation length is infinite, which, of course, is not true, e.g., existing estimates [17] indicate that $\lambda_{q}^{2} \sim$ $0.4 \mathrm{GeV}^{2}$. To study the effects due to the finite correlation length, we use a simple Gaussiantype model [14] for the coordinate dependence of the nonlocal condensates, fixing the width parameters by the estimates of the relevant operators containing an extra $D^{2}$. In particular, we take $f_{S}(\nu)=\delta\left(\nu-0.5 \lambda_{q}^{2}\right) ; f_{V}(\nu)=\delta^{\prime}\left(\nu-0.35 \lambda_{q}^{2}\right)$ for the bilocals, and $f_{1}\left(\nu_{1}, \nu_{2}, \nu_{3}\right)=$ $\delta\left(\nu_{1}-0.2 \lambda_{q}^{2}\right) \delta\left(\nu_{2}\right) \delta\left(\nu_{3}-0.2 \lambda_{q}^{2}\right) ; f_{2}\left(\nu_{1}, \nu_{2}, \nu_{3}\right)=\delta\left(\nu_{1}\right) \delta\left(\nu_{2}-0.5 \lambda_{q}^{2}\right) \delta\left(\nu_{3}-0.2 \lambda_{q}^{2}\right)$; $f_{3}\left(\nu_{1}, \nu_{2}, \nu_{3}\right)=\delta\left(\nu_{1}\right) \delta\left(\nu_{2}-0.2 \lambda_{q}^{2}\right) \delta\left(\nu_{3}-0.2 \lambda_{q}^{2}\right)$ for the trilocals.

4. $Q C D$ sum rule with nonlocal condensates. The simplest contribution is due to the $M_{\mu}$ condensate:

$$
\begin{array}{r}
\Delta \Phi_{V}\left(M^{2}, Q^{2}\right)=\frac{8 A}{81 M^{4}} \int_{0}^{1} d x \bar{x} f_{V}\left(x M^{2}\right) \exp \left(-\frac{x Q^{2}}{4 \bar{x} M^{2}}\right) \rightarrow \\
\rightarrow \frac{8 A}{81 M^{8}}\left(1+\frac{Q^{2}}{4 M^{2}\left(1-x_{0}\right)}\right) \exp \left(-\frac{x_{0} Q^{2}}{4\left(1-x_{0}\right) M^{2}}\right) .
\end{array}
$$


where $x_{0}=0.35 \lambda_{q}^{2} / M^{2}$. Just as expected, this term eventually vanishes for large $Q^{2}$, and the bigger the $\lambda_{q}^{2}$-parameter, the faster this contribution decreases with $Q^{2}$.

The largest contribution is that due to the four-quark condensate. In the factorized form it reads

$$
\begin{array}{r}
\frac{8 A}{9 M^{4}} \int_{-1}^{1} d a \int_{0}^{1} d b \int_{0}^{1} d x \int_{0}^{1} d y \theta((a-b)(x-y)) f_{S}\left(\frac{2 \bar{x} M^{2}}{1-b}\right) f_{S}\left(\frac{2 y M^{2}}{1+a}\right) \\
\frac{(a+1) \bar{b} x \bar{y}}{(a+1) \bar{b} x \bar{y}-\bar{a}(b+1) \bar{x} y} \exp \left\{-\frac{Q^{2} y(a \bar{b} x \bar{y}-\bar{a} b \bar{x} y)}{2 M^{2} \bar{y}[(a+1) \bar{b} x \bar{y}-\bar{a}(b+1) \bar{x} y]}\right\} .
\end{array}
$$

It should be noted that the argument of the exponential function is always negative in the integration region. Again, using the Gaussian ansatz, one can see that this term decreases with $Q^{2}$, and the bigger $\lambda_{q}^{2}$, the faster the decrease.

The contribution due to the trilocals has a similar, though more complicated, structure:

$$
\begin{array}{r}
\frac{4 A}{81 M^{2}} \int_{0}^{1} d a \int_{0}^{1} d b \int_{0}^{1} d x \int_{0}^{1} d y \exp \left\{-\frac{Q^{2}}{2 M^{2}}\left(\frac{\bar{a}}{2 \bar{x}(a+1)}+\frac{a}{\bar{y}(a+1)}-\frac{1}{2}\right)\right\} \\
\theta(y-x) \sum_{i=1}^{3} a_{i}\left\{\phi_{i} P_{i}\left(M^{2}, a, b, x, y\right)+\bar{\phi}_{i} S_{i}\left(M^{2}, a, b, x, y\right)\right\}
\end{array}
$$

where the $\phi$-functions are related to the quark-gluon vacuum distributions

$$
\begin{aligned}
\phi_{i} & \equiv \frac{\bar{x}}{1-a^{2}} f_{i}\left(x M^{2}, \frac{2 \bar{x} b M^{2}}{1-a}, \frac{2(y-x) M^{2}}{1+a}\right) \\
\bar{\phi}_{i} & \equiv \frac{\bar{x}}{1-a^{2}} f_{i}\left(x M^{2}, \frac{2(y-x) M^{2}}{1+a}, \frac{2 \bar{x} b M^{2}}{1-a}\right)
\end{aligned}
$$

and $P_{i}, S_{i}$ are some coefficients.

The diagrams with nonlocal gluonic condensate produce even more complicated expressions. However, in the region of intermediate $Q^{2}$, the gluonic term is numerically less significant than the quark contributions. By analogy with the quark case, we assume that the contribution due to the gluonic condensate also acquires an exponential factor $\exp \left(-\lambda_{0}^{2} Q^{2} / M^{4}\right)$.

5. Numerical results. Understanding that our models for the vacuum distributions are rather rough, we allowed the quark virtuality parameters to vary: $\lambda_{q}^{2}$ was varied between $0.4 \mathrm{GeV}^{2}$ and $0.8 \mathrm{GeV}^{2}$ and $\lambda_{g}^{2}$ varied between 0 and $1 \mathrm{GeV}^{2}$.

If $\lambda_{q}^{2}=\lambda_{g}^{2}=0$ (the local condensate approximation), then the magnitude of the parameter $s_{0}$, providing the best $M^{2}$-stability for $f_{\pi}^{2} F_{\pi}\left(Q^{2}\right)$, rapidly increases with $Q^{2}$. However, the stability region is rather narrow even for the "best" values of $s_{0}$. A very good stability, - practically a constancy of the r.h.s. of eq.(5) in the region $1 \mathrm{GeV}^{2} \lesssim M^{2} \lesssim 2.3 \mathrm{GeV}^{2}$ was observed for $\lambda_{q}^{2}=0.6 \mathrm{GeV}^{2}, 0.5 \mathrm{GeV}^{2}<\lambda_{g}^{2}<0.75 \mathrm{GeV}^{2}$ and $\lambda_{q}^{2}=0.8 \mathrm{GeV}^{2}, \lambda_{g}^{2}=0.5 \mathrm{GeV}^{2}$. Rather good stability is obtained also for $0.6 \mathrm{GeV}^{2}<\lambda_{q}^{2}<0.8 \mathrm{GeV}^{2}, \lambda_{g}^{2}=0.3 \mathrm{GeV}^{2}$.

The values for the pion local duality interval $s_{0}$ obtained from the fitting procedure are shown in Fig.1. One can see that, for the most popular value $\lambda_{q}^{2}=0.4 \mathrm{GeV}^{2}$, the increase 
of $s_{0}$ with $Q^{2}$ is much slower than in the zero virtuality case. It is even more slower for $\lambda_{a}^{2}=0.6 \mathrm{GeV}^{2}$. A remarkable fact is that if one takes $\lambda_{q}^{2}=0.8 \mathrm{GeV}^{2}$, the effective threshold $s_{0}$ is constant till $Q^{2}=10 \mathrm{GeV}^{2}$.

The $Q^{2}$-dependence of the soft contribution to the pion form factor calculated from our sum rule is demonstrated in Fig. 2. Only the results obtained with $\lambda_{q}^{2}=0.6 \mathrm{GeV}^{2}$ and $\lambda_{q}^{2}=0.8 \mathrm{GeV}^{2}$ are shown. The curves corresponding to $\lambda_{q}^{2}<0.6 \mathrm{GeV}^{2}$ go much higher than the experimental data.

Our results depend, of course, on the models we used for the nonlocal condensates. It should be emphasized, however, that our sum rule is dominated by the contribution due to the four-quark condensate $\langle\bar{q}(0) q(x) \vec{q}(y) q(z)\rangle$ approximated by the product of the simplest $\langle\bar{q}(0) q(z)\rangle$-type condensates. This approximation amounts to neglecting the dependence on the distance between the two $\bar{q} q$ pairs. With this dependence taken into account, the dominant term will fall down faster, and the $s_{0}=$ const result will be obtained for $\lambda_{q}^{2}$-values smaller than $0.8 \mathrm{GeV}^{2}$.

6. Hard gluon exchange contribution. Thus, the soft gluon contribution alone is sufficiently large to describe existing data on the pion form factor. However, one can also take into account the $O\left(\alpha_{0}\right)$ contribution to $\rho^{\text {pert }}\left(s_{1}, s_{2}, q^{2}\right)$ containing the asymptotically dominant hard gluon exchange term. The two-loop calculation is rather complicated but, as a rather accurate approximation for the local duality integral, one can use a simple formula

$$
\int_{0}^{s_{0}} d s_{1} \int_{0}^{s_{0}} d s_{2} \Delta \rho^{\text {pert }}\left(s_{1}, s_{2}, q^{2}\right) \approx \frac{\alpha_{2}}{4 \pi} \frac{s_{0}}{1+Q^{2} / 2 s_{0}}
$$

based on interpolation between the $Q^{2}=0$ value (related by the Ward identity to the $O\left(\alpha_{*}\right)$ term of the 2-point correlator) and the large- $Q^{2}$ behavior. In contrast with the asymptotic perturbative QCD formula $F_{\pi}^{\text {hard }} \sim 1 / Q^{2}$, eq.(19) contains the subasymptotic terms: $1 / Q^{2} \rightarrow 1 /\left(Q^{2}+2 s_{0}\right)$, and the resulting contribution is smaller than the asymptotic one. In fact, if one takes the "canonical" value $s_{0}=4 \pi^{2} f_{\pi}^{2} \approx 0.7 \mathrm{GeV}^{2}$, the two-loop term (19) contributes less than $0.1 \mathrm{GeV}^{2}$ to the "almost scaling" combination $Q^{2} F_{\pi}\left(Q^{2}\right)$, i.e., by factor 4 less than the soft contribution estimated under the same assumption.

The advocates of the pQCD applicability argue, that the hard-gluon exchange contribution is larger if one uses the wave function $\varphi^{C Z}(\xi)=\frac{15}{4} f_{\pi}^{2} \xi^{2}\left(1-\xi^{2}\right)$ proposed by Chernyak and A.Zhitnitsky [8] on the basis of the QCD sum rule for the moments of the pion wave function

$$
f_{\pi}^{2}\left\langle\xi^{N}\right\rangle=\frac{3 M^{2}}{4 \pi^{2}} \frac{1-e^{-\Delta_{0} / M^{2}}}{(N+1)(N+3)}+\frac{\alpha_{s}\langle G G\rangle}{12 \pi M^{2}}+\frac{16 \pi \alpha_{s}\langle\bar{q} q\rangle^{2}}{81 M^{4}}(11+4 N) .
$$

This sum rule has a striking similarity to the pion form factor sum rule (take $N \sim Q^{2} / M^{2}$ ): the perturbative term vanishes like $1 / N^{2}$ for large $N$, while the $\langle\bar{q} q\rangle$ - and $\langle G G\rangle$-terms are constant or even increasing with $N$. The ratios of the nonperturbative terms to the perturbative term grow with $N$ and, as a result, the parameters $s_{0}^{(N)}$ and the combinations $f_{\pi}^{2}\left\langle\xi^{N}\right\rangle$ straightforwardly extracted from the SR (20) are larger than the asymptotic values $s_{0}^{N=0} \approx 0.75 \mathrm{GeV}^{2}$ and $f_{\pi}^{2}\left\langle\xi^{N}\right\rangle^{a s}=3 f_{\pi}^{2} /(N+1)(N+3)$ by factors 2 (for $N=2$ ) and 3 
(for $N=4$ ). This is precisely the same effect that produced the growth of so and $F_{\star}\left(Q^{2}\right)$ for $Q^{2}>2 \mathrm{GeV}^{2}$. The large value $\left\langle\xi^{2}\right\rangle \sim 0.4$ can be attributed only to a wave function concentrated in the $\xi^{2} \sim 1$ region, and this is how the $C Z$ wave function is obtained. The crucial assumption in this derivation is that it is sufficient to take into account the lowest local condensates, which amounts to taking $\lambda_{q}^{2}=0$. However, the soft contribution calculated with $\lambda_{q}^{2}=0$ is much bigger than the data for $Q^{2} \gtrsim 2 \mathrm{GeV}^{2}$. This agrees with the observation made by Isgur and Llewellyn-Smith [10] that the use of a (three-dimensional) CZ-type wave function produces a huge soft contribution, well above the data. The hard contribution calculated in PQCD with CZ wave function is about the data, but soft and hard terms taken together are too big.

When one uses the nonlocal condensates, the parallelism between the sum rule for the pion wave function and that for the pion form factor persists: the nonperturbative terms in the modified wave function sum rule start to decrease with $N$, just like their analogues started to decrease with $Q^{2}$. Furthermore, the larger $\lambda_{q}^{2}$, the faster they decrease. If one takes $\lambda_{q}^{2}>0.4 \mathrm{GeV}^{2}$, the pion wave function extracted from the modified sum rule is much closer to the asymptotic wave function $\varphi^{a s}(\xi)=\frac{3}{4} f_{\pi}^{2}\left(1-\xi^{2}\right)$ than to the CZ one [14]. Thus, an increase in the magnitude of $\lambda_{q}^{2}$ decreases the soft contribution and, at the same time, produces the pion wave function that is more and more closer to the asymptotic wave function. As a result, the "hard" contribution also decreases. Taking $\lambda_{q}^{2}=0.6-0.8 \mathrm{GeV}^{2}$, one obtains a soft term close to data in magnitude, and a hard term that is smaller than the soft term by factor $\sim 3-5$.

7. Conclusions. Our main result in this paper is that the QCD sum rule for the pion form factor $F_{\pi}\left(Q^{2}\right)$ has a structure very similar to that of the sum rule for the moments $\left\langle\xi^{N}\right\rangle$ of the pion wave function. As a consequence, it is impossible to obtain the large value $\left\langle\xi^{2}\right\rangle \sim 0.4$, producing the $\mathrm{CZ}$ wave function, without getting a very large soft contribution to the pion form factor in the region $Q^{2} \geq 2 \mathrm{GeV}^{2}$. Using the nonlocal condensates, it is possible to reduce the soft contribution to the level dictated by experimental data. To this end, one should take a sufficiently large value $\lambda_{q}^{2} \gtrsim 0.4 \mathrm{GeV}^{2}$ for the parameter $\lambda_{q}$ characterizing average virtuality of the vacuum quarks. However, the moments of the pion wave function then also get smaller, and the resulting pion wave function cannot produce a large hard contribution. All these observations unambigously indicate that the soft contribution, corresponding to the Feynman mechanism [18], dominates the pion form factor at accessible momentum transfers $Q^{2} \lesssim 10 \mathrm{GeV}^{2}$. However, in the region $Q^{2} \sim 10 \mathrm{GeV}^{2}$ one should take into account also the suppression of the soft contribution by the Sudakov form factor of the active quark. These studies are in progress now.

Acknowledgement. We are most grateful to A.V.Efremov, B.L.Ioffe, S.V.Mikhailov, V.A.Nesterenko and I.R.Zhitnitsky for stimulating discussion. One of us (A.R.) is most grateful to N.Isgur for the warm hospitality at CEBAF where this work was completed. 


\section{References}

[1] V.L.Chernyak and A.R.Zhitnitsky, JETP Letters, 25 (1977) 510;

V.L.Chernyak, A.R.Zhitnitsky and V.G.Serbo, JETP Letters 26, (1977) 594

[2] A.V.Radyushkin, JINR preprint P2-10717, Dubna (1977)

[3] D.R.Jackson, Thesis, CALTECH (1977);

G.R.Farrar and D.R.Jackson, Phys.Rev.Lett., 43 (1979) 246

[4] A.V.Efremov and A.V.Radyushkin, Phys.Lett., 94B (1980) 245

[5] S.J.Brodsky and G.P.Lepage, Phys.Lett., 87B (1979) 359

[6] V.L.Chernyak and A.R.Zhitnitsky, Phys.Reports, 112 (1984) 173

7] S.J.Brodsky, SLAC-PUB-5116, Stanford (1989)

[8] V.L.Chernyak and A.R.Zhitnitsky, Nucl.Phys., B201 (1982) 492; B214 (1983) 547(E)

[9] A.V.Radyushkin, Acta Physica Polonica, B15 (1984) 403

[10] N.Isgur and C.H.Llewellyn Smith, Phys.Rev.Lett., 52 (1984) 1080; Phys.Lett., 217B (1989) 535

[11] B.L.Ioffe and A.V.Smilga, Phys.Lett., 114B (1982) 353

[12] V.A.Nesterenko and A.V.Radyushkin, Phys.Lett., 115B (1982) 410

[13] V.A.Nesterenko and A.V.Radyushkin, JETP Lett., 39 (1984) 707

[14] S.V.Mikhailov and A.V.Radyushkin, JETP Lett., 43 (1986) 712;

Sov.J.Nucl.Phys., 49 (1989) 494

[15] M.A.Shifman, A.I.Vainshtein and V.I.Zakharov, Nucl.Phys., B147 (1979) 385,448

[16] V.A.Nesterenko and A.V.Radyushkin, Phys.Lett.,128B (1983) 439

[17] V.M.Belyaev and B.L.Ioffe, ZhETF 93 (1982) 876;

A.A.Ovchinnikov and A.A.Pivovarov, Yad.Fiz. 48 (1988) 1135

[18] R.P.Feynman, The Photon-Hadron Interactions, W.A.Benjamin, NY (1972) 


\section{FIGURE CAPTIONS}

- Fig. 1 Dependence of the continuum threshold parameter $s_{0}$ on $Q^{2}$ for various sets of $\lambda_{q}^{2}$ and $\lambda_{g}^{2}$ :

- a) $\lambda_{q}^{2}=0, \lambda_{g}^{2}=0$

- b) $\lambda_{q}^{2}=0.4 \mathrm{GeV}^{2}, \lambda_{\sigma}^{2}=0.75 \mathrm{GeV}^{2}$;

- c) $\lambda_{q}^{2}=0.6 \mathrm{GeV}^{2}, \lambda_{g}^{2}=0.75 \mathrm{GeV}^{2}$;

- d) $\lambda_{q}^{2}=0.6 \mathrm{GeV}^{2}, \lambda_{g}^{2}=0.3 \mathrm{GeV}^{2}$;

- e) $\lambda_{q}^{2}=0.6 \mathrm{GeV}^{2}, \lambda_{g}^{2}=0.5 \mathrm{GeV}^{2}$;

- f) $\lambda_{q}^{2}=0.8 \mathrm{GeV}^{2}$.

- Fig. 2 Dependence of the combination $Q^{2} F_{\pi}\left(Q^{2}\right)$ on $Q^{2}$ for various sets of $\lambda_{q}^{2}$ and $\lambda_{g}^{2}$ :

- a) $\lambda_{q}^{2}=0.6 \mathrm{GeV}^{2}, \lambda_{g}^{2}=0.3 \mathrm{GeV}^{2}$

- b) $\lambda_{q}^{2}=0.6 \mathrm{GeV}^{2}, \lambda_{g}^{2}=0.5 \mathrm{GeV}^{2}$;

- c) $\lambda_{q}^{2}=0.8 \mathrm{GeV}^{2}, \lambda_{g}^{2}=0.3 \mathrm{GeV}^{2}$;

- d) $\lambda_{q}^{2}=0.8 \mathrm{GeV}^{2}, \lambda_{g}^{2}=0.5 \mathrm{GeV}^{2}$. 
$s_{0}$

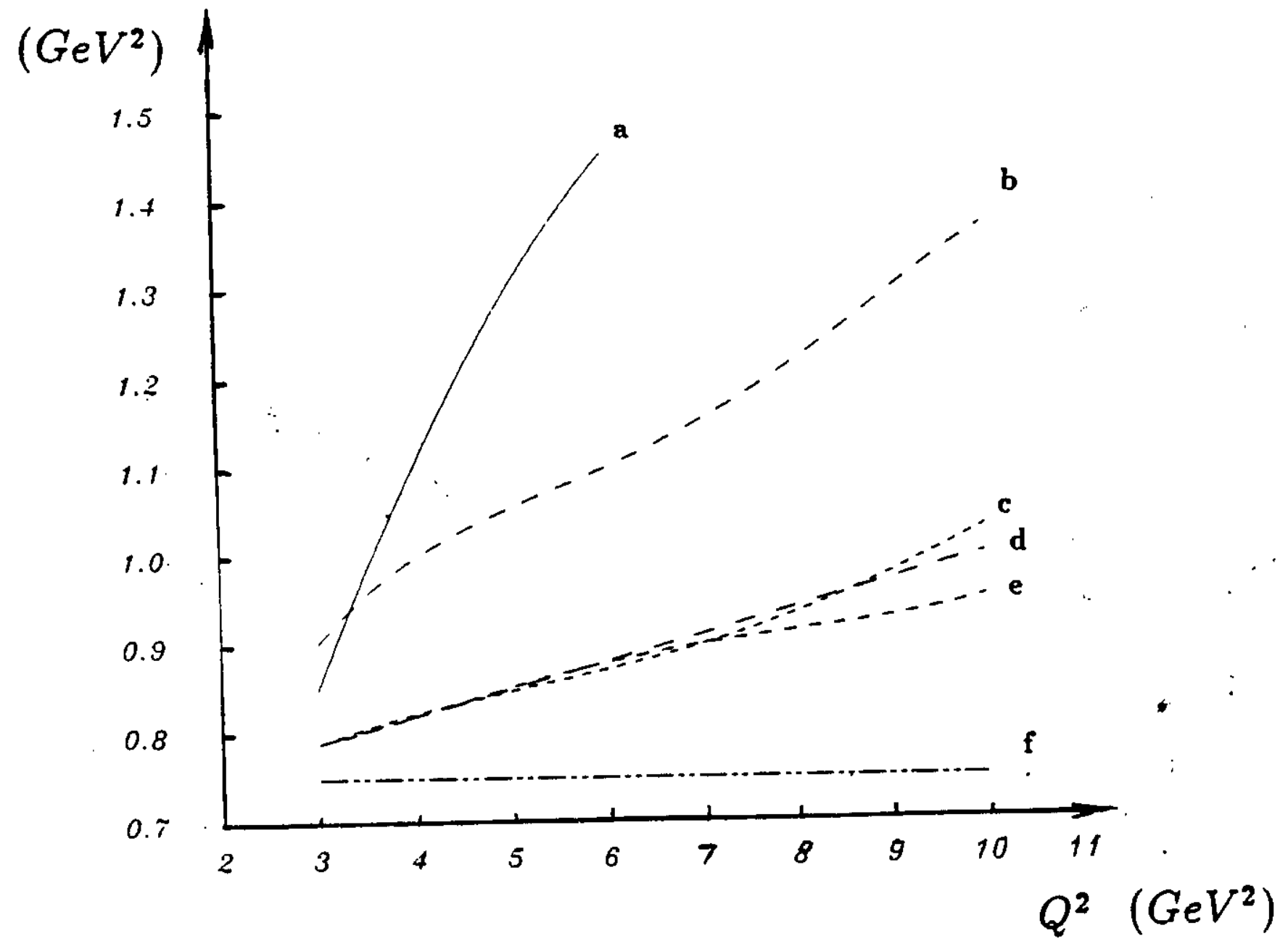

- Fig. 1 Dependence of the continuum threshold parameter so on $Q^{2}$ for various sets of $\lambda_{q}^{2}$ and $\lambda_{g}^{2}$ :

- a) $\lambda_{q}^{2}=0, \lambda_{g}^{2}=0$

- b) $\lambda_{q}^{2}=0.4 \mathrm{GeV}^{2}, \lambda_{g}^{2}=0.75 \mathrm{GeV}^{2}$;

- c) $\lambda_{q}^{2}=0.6 \mathrm{GeV}^{2}, \lambda_{g}^{2}=0.75 \mathrm{GeV}^{2}$;

- d) $\lambda_{q}^{2}=0.6 \mathrm{GeV}^{2}, \lambda_{q}^{2}=0.3 \mathrm{GeV}^{2}$;

- e) $\lambda_{q}^{2}=0.6 \mathrm{GeV}^{2}, \lambda_{g}^{2}=0.5 \mathrm{GeV}^{2}$;

-f) $\lambda_{q}^{2}=0.8 \mathrm{GeV}^{2}$. 
